\title{
Infinitely many sign-changing solutions for $p$-Laplacian equation involving the critical Sobolev exponent
}

Yuanze $\mathrm{Wu}^{*}$ and Yisheng Huang

\section{"Correspondence:}

wuyuanzesuda@126.com Department of Mathematics, Soochow University, Suzhou, Jiangsu, 215006, P.R. China

\section{Abstract}

In this paper, we study the following problem:

$$
\left\{\begin{array}{l}
-\Delta_{p} u=\lambda|u|^{p-2} u+|u|^{p^{*}-2} u \text { in } \Omega \\
u=0 \quad \text { on } \partial \Omega
\end{array}\right.
$$

where $\Omega \subset \mathbb{R}^{N}$ is a smooth bounded domain, $1<p<N,-\Delta_{p} u=\operatorname{div}\left(|\nabla u|^{p-2} \nabla u\right)$ is the $p$-Laplacian, $p^{*}=p N /(N-p)$ is the critical Sobolev exponent and $\lambda>0$ is a parameter. By establishing a new deformation lemma, we show that if $N>p^{2}+p$, then for each $\lambda>0$, this problem has infinitely many sign-changing solutions, which extends the results obtained in (Cao et al. in J. Funct. Anal. 262: 2861-2902, 2012; Schechter and Zou in Arch. Ration. Mech. Anal. 197: 337-356, 2010).

\section{Introduction}

In this paper, we consider the following problem:

$$
\left\{\begin{array}{l}
-\Delta_{p} u=\lambda|u|^{p-2} u+|u|^{p^{*}-2} u \quad \text { in } \Omega \\
u=0 \quad \text { on } \partial \Omega
\end{array}\right.
$$

where $\Omega \subset \mathbb{R}^{N}(N \geq 3)$ is a smooth bounded domain, $1<p<N,-\Delta_{p} u=\operatorname{div}\left(|\nabla u|^{p-2} \nabla u\right)$ is the $p$-Laplacian, $p^{*}=p N /(N-p)$ is the critical Sobolev exponent and $\lambda>0$ is a parameter.

The first existence result of Problem (1.1) with $p=2$ was obtained by Brezis and Nirenberg in the celebrated paper [1]. In that paper, the authors proved that Problem (1.1) had a positive solution for $N \geq 4$ and $\lambda \in\left(0, \lambda_{1}^{*}\right)$ or $N=3$ and $\lambda \in\left(\lambda_{1}^{*} / 4, \lambda_{1}^{*}\right)$, where $\lambda_{1}^{*}$ is the first eigenvalue of $\left(-\Delta, H_{0}^{1}(\Omega)\right)$. After that, many existence results have appeared for (1.1); one can see, for example, [2-7] and the references therein for case of $p=2$ and [8-11] and the references therein for case of $1<p<N$. In particular, in the case of $p=2$, the authors in [2] proved that the number of solutions of Problem (1.1) is bounded below by the number of eigenvalues of $\left(-\Delta, H_{0}^{1}(\Omega)\right)$ lying in the open interval $\left(\lambda, \lambda+S|\Omega|^{-2 / N}\right)$, where $S$ is the best Sobolev constant and $|\Omega|$ is the Lebesgue measure of $\Omega$. In [5], the existence of infinitely many sign-changing solutions of (1.1) with $p=2$ has been obtained when $N \geq 4$, $\lambda>0$ and $\Omega$ is a ball, while it has been shown in [6] that (1.1) with $p=2$ has infinitely many 
sign-changing radial solutions when $N \geq 7, \lambda>0$ and $\Omega$ also is a ball. We remark that the methods used in $[5,6]$ are strongly dependent on the symmetry of the ball $\Omega$. For a general bounded smooth domain $\Omega$, by the method of invariant sets of the descending flow, the authors in [7] have shown that (1.1) with $p=2$ has infinitely many sign-changing solutions when $N \geq 7$ and $\lambda>0$, which extends the main result in [4].

The main purpose of this paper is to try to obtain the existence of infinitely many signchanging solutions of Problem (1.1) for general $p \in(1, N)$. In a very recent work [9], the authors have proved that (1.1) has infinitely many solutions for $\lambda>0$ and $N>p^{2}+p$. However, by using the Picone identity $(c f .[12,13])$, we see that every nonzero solution of Problem (1.1) is sign-changing for $\lambda \geq \lambda_{1}$, where $\lambda_{1}$ is the first eigenvalue of $\left(-\Delta_{p}, W_{0}^{1, p}(\Omega)\right)$ (see Lemma 2.1 for more details). Hence, to achieve our purpose, we mainly consider the situation of $\lambda \in\left(0, \lambda_{1}\right)$.

Our main result in this paper is the following.

Theorem 1.1 Assume that $N>p^{2}+p$ and $\lambda>0$. Then Problem (1.1) has infinitely many sign-changing solutions.

Since $p^{*}$ is the critical Sobolev exponent, in order to overcome the lack of compactness of the embedding of $W_{0}^{1, p}(\Omega)$ in the Lebesgue space $L^{p^{*}}(\Omega)$, we follow the ideas of $[4,7,9]$ to consider the following auxiliary problems:

$$
\left\{\begin{array}{l}
-\Delta_{p} u=\lambda|u|^{p-2} u+|u|^{p_{n}-2} u \text { in } \Omega \\
u=0 \text { on } \partial \Omega
\end{array}\right.
$$

where $p_{n}<p^{*}$ and $p_{n}$ is increasing to $p^{*}$. It has been shown by [14, Theorem 1.2] that for every $n$, Problem $\left(\mathcal{P}_{n}\right)$ has infinitely many sign-changing solutions $\left\{u_{n, k}\right\}_{k \in \mathbb{N}}$. Hence, to prove Theorem 1.1, we will show that for every $k \in \mathbb{N},\left\{u_{n, k}\right\}$ converges to some signchanging solution $u_{k}$ of (1.1) as $n \rightarrow \infty$, and that $\left\{u_{k}\right\}$ are different. The convergence of $\left\{u_{n, k}\right\}$ can be done with the help of [9, Theorem 1.2], which we show in Lemma 2.3. To distinguish $\left\{u_{k}\right\}$, we shall establish a new deformation lemma on special sets in $W_{0}^{1, p}(\Omega)$; see Lemma 2.5 for details.

Throughout this paper, we will always respectively denote $\|u\|$ and $\|u\|_{r}$ by the usual norm in spaces $W_{0}^{1, p}(\Omega)$ and $L^{r}(\Omega)(r \geq 1)$. Let $C$ be indiscriminately used to denote various positive constants.

\section{Proof of Theorem 1.1}

We first consider the case of $\lambda \geq \lambda_{1}$. Recall that $\lambda_{1}$, the first eigenvalue of $-\Delta_{p}$ in $W_{0}^{1, p}(\Omega)$, given by $\lambda_{1}:=\inf \left\{\int_{\Omega}|\nabla u|^{p} d x, \int_{\Omega}|u|^{p} d x=1\right\}$, is simple and there exists a positive eigenfunction $e_{1} \in W_{0}^{1, p}(\Omega)$ corresponding to $\lambda_{1}$ such that $\int_{\Omega}\left|\nabla e_{1}\right|^{p-2} \nabla e_{1} \nabla \eta d x=\lambda_{1} \int_{\Omega} e_{1}^{p-1} \eta d x$ for every $\eta \in W_{0}^{1, p}(\Omega)$ (cf. [15]). Moreover, by [16, Proposition 2.1], we know that $e_{1} \in$ $L^{\infty}(\Omega) \cap C_{\text {loc }}^{1, \alpha}(\Omega)$. On the other hand, we have the following proposition which is the socalled Picone identity. 
Proposition 2.1 [13, Lemma A.6] Let $u, v \in W_{\mathrm{loc}}^{1, p}(\Omega) \cap C(\Omega)$ be such that $u \geq 0, v>0$ and $\frac{u}{v} \in W_{\text {loc }}^{1, p}(\Omega)$. Then

$$
\begin{aligned}
\int_{\Omega} & \nabla\left(\frac{u^{p}}{v^{p-1}}\right)|\nabla v|^{p-2} \nabla v d x \\
\quad= & \int_{\Omega}\left(p\left(\frac{u}{v}\right)^{p-1}|\nabla v|^{p-2} \nabla v \nabla u-(p-1)\left(\frac{u}{v}\right)^{p}|\nabla v|^{p}\right) d x .
\end{aligned}
$$

Moreover,

$$
\int_{\Omega}\left(p\left(\frac{u}{v}\right)^{p-1}|\nabla v|^{p-2} \nabla v \nabla u-(p-1)\left(\frac{u}{v}\right)^{p}|\nabla v|^{p}\right) d x \leq \int_{\Omega}|\nabla u|^{p} d x
$$

and the equality holds if and only if $u=c v$ for some constant $c>0$.

Lemma 2.1 Assume that $u \in W_{0}^{1, p}(\Omega)$ is a nonzero solution of (1.1) for $\lambda \geq \lambda_{1}$. Then $u$ is sign-changing.

Proof By a contradiction, we may assume $u \geq 0$. By using a standard regularity argument and [17, Lemmas 3.2 and 3.3], we have $u \in C^{1, \alpha}(\Omega)$ for some $\alpha \in(0,1)$. Thus, it follows from the strong maximum principle (cf. [18]) that $u>0$. Now, for every $\varepsilon>0$, by applying the above Picone identity (i.e., Proposition 2.1) to $u+\varepsilon$ and $e_{1}$, we see

$$
\int_{\Omega}\left|\nabla e_{1}\right|^{p} d x \geq \int_{\Omega} \nabla\left(\frac{e_{1}^{p}}{(u+\varepsilon)^{p-1}}\right)|\nabla u|^{p-2} \nabla u d x
$$

Noting that $u$ is a solution of (1.1), we have

$$
\int_{\Omega}\left|\nabla e_{1}\right|^{p} d x \geq \int_{\Omega}\left(\lambda \frac{u^{p-1}}{(u+\varepsilon)^{p-1}}+\frac{u^{p^{*}-1}}{(u+\varepsilon)^{p-1}}\right) e_{1}^{p} d x
$$

It follows from the Fatou lemma that

$$
\begin{aligned}
\int_{\Omega}\left|\nabla e_{1}\right|^{p} d x & \geq \liminf _{\varepsilon \rightarrow 0} \int_{\Omega}\left(\lambda \frac{u^{p-1}}{(u+\varepsilon)^{p-1}}+\frac{u^{p^{*}-1}}{(u+\varepsilon)^{p-1}}\right) e_{1}^{p} d x \\
& \geq \int_{\Omega}\left(\lambda+u^{p^{*}-p}\right) e_{1}^{p} d x
\end{aligned}
$$

which is impossible since $\int_{\Omega}\left|\nabla e_{1}\right|^{p} d x=\lambda_{1} \int_{\Omega} e_{1}^{p}, u>0, e_{1}>0$ and $\lambda \geq \lambda_{1}$. Therefore, we have proved Lemma 2.1 .

Next, we consider the case of $\lambda<\lambda_{1}$.

It is clear that the corresponding functional of $\left(\mathcal{P}_{n}\right) I_{n}: W_{0}^{1, p}(\Omega) \rightarrow \mathbb{R}$, given by

$$
I_{n}(u)=\frac{1}{p}\left(\|u\|^{p}-\lambda\|u\|_{p}^{p}\right)-\frac{1}{p_{n}}\|u\|_{p_{n}}^{p_{n}}
$$

is $C^{1}$ Fréchet differentiable. Let $X_{m}=\operatorname{span}\left\{\varphi_{1}, \ldots, \varphi_{m}\right\}$, where $\left\{\varphi_{i}\right\}$ is a linearly independent sequence of $W_{0}^{1, p}(\Omega)$. It is easy to show that there exists $R_{m}>0$ such that $I_{n}(u) \leq-1$ for 
$u \in X_{m} \backslash B_{m}$, where $B_{m}:=\left\{u \in X_{m}:\|u\| \leq R_{m}\right\}$ (cf. [14, Lemma 3.9]). We denote

$$
\begin{aligned}
& P(-P):=\left\{u \in W_{0}^{1, p}(\Omega): u \geq 0(u \leq 0) \text { a.e. }\right\}, \\
& D_{\mu}^{ \pm}:=\left\{u \in W_{0}^{1, p}(\Omega): \operatorname{dist}(u, \pm P) \leq \mu\right\}, \quad D_{\mu}:=D_{\mu}^{+} \cup D_{\mu}^{-}, \\
& G_{m}:=\left\{h \in C\left(B_{m}, W_{0}^{1, p}(\Omega)\right): h \text { is odd, } h(x)=x \text { for } x \in \partial_{X_{m}} B_{m}\right\} .
\end{aligned}
$$

Recall that the genus of a symmetric set $A$ of $W_{0}^{1, p}(\Omega)$ is defined by

$$
\operatorname{gen}(A):=\inf \left\{k \geq 0: \exists f \in C\left(A, \mathbb{R}^{k} \backslash\{0\}\right), f \text { is odd }\right\}
$$

Here, we say that $A$ is symmetric if $x \in A$ implies $-x \in A$.

By [14, Theorem 1.2], we know that, for every $n \in \mathbb{N}, I_{n}(u)$ has infinitely many critical points, denoted by $\left\{u_{n, k}\right\}_{k \in \mathbb{N}}$, in $X \backslash D_{\mu}$ for $\mu$ small enough. Moreover,

$$
I_{n}\left(u_{n, k}\right)=d_{n, k}:=\inf _{Z \in \Gamma_{k}} \sup _{u \in Z} I_{n}(u)
$$

where $\Gamma_{k}:=\left\{h\left(B_{m} \backslash B\right) \backslash D_{\mu}: h \in G_{m}\right.$ for $m \geq n, B=-B \subset B_{m}$ open, gen $\left.(B) \leq m-n\right\}$.

Lemma 2.2 For every $k \in \mathbb{N}$, there exists $d_{k}^{*}>0$ such that $\left\|u_{n, k}\right\| \leq d_{k}^{*}$ for all $n \in \mathbb{N}$.

Proof Consider the following auxiliary functional:

$$
I_{*}(u):=\frac{1}{p}\left(\|u\|^{p}-\|u\|_{p}^{p}\right)-\frac{1}{p^{*}}\|u\|_{\sigma}^{\sigma}
$$

where $\sigma=\left(p+p^{*}\right) / 2$. Since $p_{n} \rightarrow p^{*}$, we may assume $p_{n}>\sigma$ for all $n \in \mathbb{N}$. Then $\frac{1}{p^{*}}\|u\|_{\sigma}^{\sigma} \leq$ $\frac{\operatorname{meas}(\Omega)}{p^{*}}+\frac{1}{p_{n}}\|u\|_{p_{n}}^{p_{n}}$ for all $n \in \mathbb{N}$. This means

$$
I_{*}(u)=I_{n}(u)+\left(\frac{1}{p_{n}}\|u\|_{p_{n}}^{p_{n}}-\frac{1}{p^{*}}\|u\|_{\sigma}^{\sigma}\right) \geq I_{n}(u)-\frac{\operatorname{meas}(\Omega)}{p^{*}} .
$$

Note that $I_{*}(u)$ is the corresponding functional of the following equation:

$$
\left\{\begin{array}{l}
-\Delta_{p} u=\lambda|u|^{p-2} u+\frac{\sigma}{p^{*}}|u|^{\sigma-2} u \quad \text { in } \Omega \\
u=0 \text { on } \partial \Omega,
\end{array}\right.
$$

and the nonlinearity satisfies the assumptions of [14, Theorem 1.2]. Thus, this equation has a sequence of solutions $\left\{v_{k}\right\} \subset W_{0}^{1, p}(\Omega) \backslash\left(D_{\mu}^{+} \cup D_{\mu}^{-}\right)$such that

$$
I_{*}\left(v_{k}\right)=\bar{d}_{k}:=\inf _{Z \in \Gamma_{k}} \sup _{u \in Z} I_{*}(u)
$$

for $\mu$ small enough. For every $k \in \mathbb{N}$, the definitions of $\bar{d}_{k}$ and $d_{k, n}$, together with (2.2), imply $\bar{d}_{k}+\frac{\text { meas }(\Omega)}{p^{*}} \geq d_{k, n}$ for all $n \in \mathbb{N}$. On the other hand, since for every $n,\left\{u_{n, k}\right\}_{k \in \mathbb{N}}$ is a sequence of solutions for $\left(\mathcal{P}_{n}\right)$ whose energies satisfy $(2.1)$, it follows that $d_{n, k} \geq\left(\frac{1}{p}-\frac{1}{p_{1}}\right)(1-$ $\left.\frac{\lambda}{\lambda_{1}}\right)\left\|u_{n, k}\right\|^{p}$. We complete the proof by choosing $d_{k}^{*}=\left(\frac{\left(\bar{d}_{k} p^{*}+\operatorname{meas}(\Omega)\right) p_{1} p_{1}}{p^{*}\left(p_{1}-p\right)\left(\lambda_{1}-\lambda\right)}\right)^{1 / p}$. 
By Lemma 2.2 and [9, Theorem 1.2], we know that for each $k \in \mathbb{N}$, there exists $u_{k} \in$ $W_{0}^{1, p}(\Omega)$ such that $u_{n, k} \rightarrow u_{k}$ as $n \rightarrow \infty$ in $W_{0}^{1, p}(\Omega)$. The next lemma will give more information about $u_{k}$.

Lemma 2.3 $u_{k}$ is a sign-changing solution of Problem (1.1) for every $k \in \mathbb{N}$.

Proof We first prove that $u_{k}$ is a solution of Problem (1.1) for every $k \in \mathbb{N}$. Since $u_{n, k} \rightarrow u_{k}$ as $n \rightarrow \infty$ in $W_{0}^{1, p}(\Omega)$,

$$
\int_{\Omega}\left|\nabla u_{n, k}\right|^{p-2} \nabla u_{n, k} \nabla \varphi d x \rightarrow \int_{\Omega}\left|\nabla u_{k}\right|^{p-2} \nabla u_{k} \nabla \varphi d x
$$

and

$$
\int_{\Omega}\left|u_{n, k}\right|^{p-2} u_{n, k} \varphi d x \rightarrow \int_{\Omega}\left|u_{k}\right|^{p-2} u_{k} \varphi d x
$$

as $n \rightarrow \infty$ for every $\varphi \in W_{0}^{1, p}(\Omega)$. If we can prove

$$
\int_{\Omega}\left|u_{n, k}\right|^{p_{n}-2} u_{n, k} \varphi d x \rightarrow \int_{\Omega}\left|u_{k}\right|^{2^{*}-2} u_{k} \varphi d x
$$

as $n \rightarrow \infty$ for every $\varphi \in W_{0}^{1, p}(\Omega)$, then $u_{k}$ is a solution of (1.1) for $u_{n, k}$ is a solution of $\left(\mathcal{P}_{n}\right)$. Indeed, $u_{n, k} \rightarrow u_{k}$ a.e. in $\Omega$ as $n \rightarrow \infty$ since $u_{n, k} \rightarrow u_{k}$ in $W_{0}^{1, p}(\Omega)$. By the Egoroff theorem, for every $\delta>0$, there exists $\Omega_{\delta}$ such that $u_{n, k} \rightarrow u_{k}$ uniformly in $\Omega \backslash \Omega_{\delta}$ and $\left|\Omega_{\delta}\right|<$ $\delta$, where $\left|\Omega_{\delta}\right|$ is the Lebesgue measure of $\Omega_{\delta}$. This, together with the Lebesgue dominated convergence theorem, implies

$$
\lim _{n \rightarrow \infty} \int_{\Omega \backslash \Omega_{\delta}}\left|u_{n, k}\right|^{p_{n}-2} u_{n, k} \varphi d x=\int_{\Omega \backslash \Omega_{\delta}}\left|u_{k}\right|^{p^{*}-2} u_{k} \varphi d x \quad \text { for every } \varphi \in W_{0}^{1, p}(\Omega) .
$$

On the other hand, for every $\varphi \in W_{0}^{1, p}(\Omega)$, we have

$$
\begin{aligned}
&\left.\int_{\Omega_{\delta}}|| u_{n, k}\right|^{p_{n}-2} u_{n, k}-\left|u_{k}\right|^{p^{*}-2} u_{k}|| \varphi \mid d x \\
& \leq\left.\int_{\Omega_{\delta}}|| u_{n, k}\right|^{p_{n}-2} u_{n, k}-\left|u_{n, k}\right|^{p-1}-\left|u_{n, k}\right|^{p^{*}-1}|| \varphi \mid d x \\
& \quad+\left.\int_{\Omega_{\delta}}|| u_{k}\right|^{p-1}+\left|u_{k}\right|^{p^{*}-1}-\left|u_{k}\right|^{p^{*}-2} u_{k}|| \varphi \mid d x \\
& \quad+\left.\int_{\Omega_{\delta}}|| u_{k}\right|^{p-1}+\left|u_{k}\right|^{p^{*}-1}-\left|u_{n, k}\right|^{p-1}-\left|u_{n, k}\right|^{p^{*}-1}|| \varphi \mid d x \\
& \leq\left. 2 \int_{\Omega_{\delta}}|| u_{n, k}\right|^{p-1}+\left|u_{n, k}\right|^{p^{*}-1}|| \varphi\left|d x+\int_{\Omega_{\delta}}\right|\left|u_{k}\right|^{p-1}+\left|u_{k}\right|^{p^{*}-1}-\left|u_{k}\right|^{p^{*}-2} u_{k}|| \varphi \mid d x \\
& \quad+\left.\int_{\Omega_{\delta}}|| u_{k}\right|^{p-1}+\left|u_{k}\right|^{p^{*}-1}-\left|u_{n, k}\right|^{p-1}-\left|u_{n, k}\right|^{p^{*}-1}|| \varphi \mid d x \\
& \leq\left.2 \int_{\Omega_{\delta}}|| u_{k}\right|^{p-1}+\left|u_{k}\right|^{p^{*}-1}|| \varphi\left|d x+\int_{\Omega_{\delta}}\right|\left|u_{k}\right|^{p-1}+\left|u_{k}\right|^{p^{*}-1}-\left|u_{k}\right|^{p^{*}-2} u_{k}|| \varphi \mid d x \\
&+\left.3 \int_{\Omega_{\delta}}|| u_{k}\right|^{p-1}+\left|u_{k}\right|^{p^{*}-1}-\left|u_{n, k}\right|^{p-1}-\left|u_{n, k}\right|^{p^{*}-1}|| \varphi \mid d x .
\end{aligned}
$$


For every $\varepsilon>0$, by the above inequality and the absolute continuity of the integral, we can take $\delta$ small enough such that

$$
\left.2 \int_{\Omega_{\delta}}|| u_{k}\right|^{p-1}+\left|u_{k}\right|^{p^{*}-1}|| \varphi\left|d x+\int_{\Omega_{\delta}}\right|\left|u_{k}\right|^{p-1}+\left|u_{k}\right|^{p^{*}-1}-\left|u_{k}\right|^{p^{*}-2} u_{k}|| \varphi \mid d x<\varepsilon / 3 .
$$

For this $\delta$, since $u_{n, k} \rightarrow u_{k}$ in $W_{0}^{1, p}(\Omega)$,

$$
\left.3 \int_{\Omega_{\delta}}|| u_{k}\right|^{p-1}+\left|u_{k}\right|^{p^{*}-1}-\left|u_{n, k}\right|^{p-1}-\left|u_{n, k}\right|^{p^{*}-1}|| \varphi \mid d x<\varepsilon / 3
$$

for $n$ large enough. By (2.4), for this $\delta$, we have

$$
\left.\int_{\Omega \backslash \Omega_{\delta}}|| u_{n, k}\right|^{p_{n}-2} u_{n, k} \varphi-\left|u_{k}\right|^{p^{*}-2} u_{k} \varphi \mid d x<\varepsilon / 3
$$

for $n$ large enough. So (2.3) holds. Moreover, by a similar proof, we can show $d_{k}:=$ $\lim _{n \rightarrow \infty} d_{n, k}=I_{n}\left(u_{n, k}\right)=I\left(u_{k}\right)$.

Next, we will show $u_{k}$ is sign-changing for all $k \in \mathbb{N}$. Since for each $n \in \mathbb{N}, u_{n, k}$ is a signchanging solution of $\left(\mathcal{P}_{n}\right)$, multiplying $\left(\mathcal{P}_{n}\right)$ by $u_{n, k}^{ \pm}$, we obtain $\left\|u_{n, k}^{ \pm}\right\|^{p}=\lambda\left\|u_{n, k}^{ \pm}\right\|_{p}^{p}+\left\|u_{n, k}^{ \pm}\right\|_{p_{n}}^{p_{n}}$, where $u^{ \pm}=\max \{ \pm u, 0\}$. Note that $\lambda<\lambda_{1}$, by the Sobolev imbedding theorem, we have $0<\left(1-\frac{\lambda}{\lambda_{1}}\right) C \leq\left\|u_{n, k}^{ \pm}\right\|_{p^{*}}^{p_{n}-p}$. It follows that $u_{n, k} \rightarrow u_{k}$ in $L^{p^{*}}(\Omega)$ as $n \rightarrow \infty$ for $u_{n, k} \rightarrow u_{k}$ in $W_{0}^{1, p}(\Omega)$ as $n \rightarrow \infty$. This gives $0<\left(1-\frac{\lambda}{\lambda_{1}}\right) C \leq\left\|u_{k}^{ \pm}\right\|_{p^{*}}^{p^{*}-p}$, i.e., $u_{k}^{ \pm} \neq 0$ for all $k \in \mathbb{N}$.

Let $\varepsilon>0$ and $c \in \mathbb{R}$, we denote

$$
\begin{aligned}
& K:=\left\{u \in W_{0}^{1, p}(\Omega): I^{\prime}(u)=0\right\}, \quad K_{c}:=\left\{u \in W_{0}^{1, p}(\Omega): I(u)=c, I^{\prime}(u)=0\right\}, \\
& K_{\mu}^{*}:=K \backslash\left(\operatorname{int}\left(D_{\mu}^{+}\right) \cup \operatorname{int}\left(D_{\mu}^{-}\right)\right), \quad K_{c, \mu}^{*}:=K_{c} \backslash\left(\operatorname{int}\left(D_{\mu}^{+}\right) \cup \operatorname{int}\left(D_{\mu}^{-}\right)\right), \\
& \mathcal{N}_{c, \mu, \varepsilon}:=\left\{u \in W_{0}^{1, p}(\Omega): \operatorname{dist}\left(u, K_{c}^{*}\right)<\varepsilon\right\} .
\end{aligned}
$$

Thanks to Lemma 2.3, $u_{k} \in K_{\mu_{k}}^{*}$ for some $\mu_{k}>0$. We claim that $\left\{u_{k}\right\} \subset K_{\mu}^{*}$ for some $\mu>0$. Indeed, if not, then $\operatorname{dist}\left(u_{k}, P\right) \rightarrow 0$ as $k \rightarrow \infty$ without loss of generality. On the one hand, since $u_{k}$ is a solution of (1.1), $\left\langle I^{\prime}\left(u_{k}\right), u_{k}-S_{\lambda}\left(u_{k}\right)\right\rangle_{W_{0}^{1, p}(\Omega), W_{0}^{-1, p}(\Omega)}=0$, where $S_{\lambda}\left(u_{k}\right):\left(-\Delta_{p}\right)^{-1}\left(\lambda\left|u_{k}\right|^{p-2} u_{k}+\left|u_{k}\right|^{p^{*}-2} u_{k}\right)$. On the other hand, by [17, Lemma 3.7], we have

$$
\left\langle I^{\prime}\left(u_{k}\right), u_{k}-\left.S_{\lambda}\left(u_{k}\right)\right|_{W_{0}^{1, p}(\Omega), W_{0}^{-1, p}(\Omega)} \geq C\left\|u_{k}-S_{\lambda}\left(u_{k}\right)\right\|^{2}\left(\left\|u_{k}\right\|+\left\|S_{\lambda}\left(u_{k}\right)\right\|\right)^{p-2}\right.
$$

for $1<p<2$ and

$$
\left\langle I^{\prime}\left(u_{k}\right), u_{k}-\left.S_{\lambda}\left(u_{k}\right)\right|_{W_{0}^{1, p}(\Omega), W_{0}^{-1, p}(\Omega)} \geq C\left\|u_{k}-S_{\lambda}\left(u_{k}\right)\right\|^{p}\right.
$$

for $p \geq 2$. Note that by a similar proof of [14, Lemma 3.3], we can see that $S_{\lambda}\left(D_{\mu}^{ \pm}\right) \subset \operatorname{int}\left(D_{\mu}^{ \pm}\right)$ for $\mu$ small enough. Thus, $\left\|u_{k}-S_{\lambda}\left(u_{k}\right)\right\|>0$ for $k$ large enough. This implies

$$
\left\langle I^{\prime}\left(u_{k}\right), u_{k}-S_{\lambda}\left(u_{k}\right)\right\rangle_{W_{0}^{1, p}(\Omega), W_{0}^{-1, p}(\Omega)} \geq C_{k}>0
$$

for $k$ large enough, which contradicts $\left\langle I^{\prime}\left(u_{k}\right), u_{k}-S_{\lambda}\left(u_{k}\right)\right\rangle_{W_{0}^{1, p}(\Omega), W_{0}^{-1, p}(\Omega)}=0$. For the sake of convenience, we denote $K_{\mu}^{*}, K_{c, \mu}^{*}, \mathcal{N}_{c, \mu, \varepsilon}$ by $K^{*}, K_{c}^{*}, \mathcal{N}_{c, \varepsilon}$. Note that for every $c \in \mathbb{R}, K_{c}$ is 
compact in $W_{0}^{1, p}(\Omega)(c f$. [9, Theorem 1.2]). It follows from [19, Proposition 7.5] that there exists $\varepsilon>0$ such that

$$
\operatorname{gen}\left(\mathcal{N}_{c, 2 \varepsilon}\right)=\operatorname{gen}\left(K_{c}^{*}\right)<+\infty .
$$

Let $J_{n}^{c}:=\left\{u \in W_{0}^{1, p}(\Omega): I_{n}(u) \leq c\right\}$ and $\mathcal{Q}_{n}^{c}:=D_{\mu} \cup J_{n}^{c}$. Let $J^{c}:=\left\{u \in W_{0}^{1, p}(\Omega): I(u) \leq c\right\}$. For $\delta>0$ small enough, we define $\mathcal{A}_{n, \varepsilon}^{c, \delta}:=\left(\mathcal{Q}_{n}^{c+\delta} \backslash \mathcal{Q}_{n}^{c-\delta}\right) \backslash \mathcal{N}_{c, \varepsilon}$, then we have the following.

Lemma 2.4 Assume that there exists $\delta>0$ such that $K^{*} \cap J^{c+\delta} \backslash \operatorname{int}\left(J^{c-\delta}\right)=K_{c}^{*}$ for $n$ large. Then there exists $\gamma>0$ such that $\left\|I_{n}^{\prime}(u)\right\| \geq \gamma$ for $u \in \mathcal{A}_{n, \varepsilon}^{c, \delta}$ and large $n$.

Proof Assume a contradiction. Then, for every $n \in \mathbb{N}$, there exists $\left\{v_{n, k}\right\} \subset \mathcal{A}_{n, \varepsilon}^{c, \delta}$ such that $\lim _{k \rightarrow \infty} I_{n}^{\prime}\left(v_{n, k}\right)=0$. It is clear that $I_{n}$ satisfies the (PS) condition for every $n \in \mathbb{N}$. Hence there exists $v_{n} \in W_{0}^{1, p}(\Omega)$ such that, up to a subsequence, $v_{n, k} \rightarrow v_{n}$ in $W_{0}^{1, p}(\Omega)$ as $k \rightarrow \infty$ with $I_{n}^{\prime}\left(v_{n}\right)=0$ and $I_{n}\left(v_{n}\right) \in[c-\delta, c+\delta]$. This implies

$$
c+\delta \geq I_{n}\left(v_{n}\right)=\left(\frac{1}{p}-\frac{1}{p_{n}}\right)\left(1-\frac{\lambda}{\lambda_{1}}\right)\left\|v_{n}\right\|^{p} \geq\left(\frac{1}{p}-\frac{1}{p_{1}}\right)\left(1-\frac{\lambda}{\lambda_{1}}\right)\left\|v_{n}\right\|^{p} .
$$

Thus, by [9, Theorem 1.2], up to a subsequence, we see that there exists $v_{0} \in W_{0}^{1, p}(\Omega)$ such that $v_{n} \rightarrow v_{0}$ in $W_{0}^{1, p}(\Omega)$ as $n \rightarrow \infty$. Moreover, by using the arguments in the proof of Lemma 2.3, we have $I^{\prime}\left(v_{0}\right)=0$ and $I\left(v_{0}\right) \in[c-\delta, c+\delta]$. On the other hand, for large $n$, $v_{n} \notin\left(\operatorname{int}\left(D_{\mu}^{+}\right) \cup \operatorname{int}\left(D_{\mu}^{-}\right)\right) \cup \mathcal{N}_{c, \varepsilon}$ since $v_{n, k} \in \mathcal{A}_{n, \varepsilon}^{c, \delta}$. It follows that $v_{0} \notin\left(\operatorname{int}\left(D_{\mu}^{+}\right) \cup \operatorname{int}\left(D_{\mu}^{-}\right)\right) \cup \mathcal{N}_{c, \varepsilon}$. This contradicts the fact that $K^{*} \cap J_{n}^{c+\delta} \backslash \operatorname{int}\left(J_{n}^{c-\delta}\right)=K_{c}^{*}$.

Lemma 2.5 Assume that there exists $\gamma>0$ such that $\left\|I_{n}^{\prime}(u)\right\| \geq \gamma$ for every $u \in \mathcal{A}_{n, \varepsilon}^{c, \delta}$ and large n. Then there exist $\delta>0$ and an odd continuous map $\eta_{n}$ such that $\eta_{n}: \mathcal{A}_{n, 2 \varepsilon}^{c, \delta} \cup \mathcal{Q}_{n}^{c-\delta} \rightarrow$ $\mathcal{Q}_{n}^{c-\delta}$ and $\left.\eta\right|_{\mathcal{Q}_{n}^{c-\delta}}=$ Id for large $n$.

Proof We first assume $1<p<2$. It is clear that there exists $L>0$ such that

$$
\|u\|+\left\|S_{n, \lambda}(u)\right\| \leq L \quad \text { for all } u \in \mathcal{N}_{c, 2 \varepsilon}
$$

where

$$
\left\langle S_{n, \lambda}(u), \varphi\right\rangle:=\int_{\Omega}\left(\lambda|u|^{p-2} u+|u|^{p_{n}-2} u\right) \varphi d x \quad \text { for } u \in W_{0}^{1, p}(\Omega) \text { and } \varphi \in W_{0}^{-1, p}(\Omega) .
$$

Let $T_{n, \lambda}: W_{0}^{1, p}(\Omega) \backslash K \rightarrow W_{0}^{1, p}(\Omega)$ be the local Lipschitz continuous operator obtained in [14, Lemma 2.1] and let $\phi_{u}(t)$ be the solution of the following O.D.E.

$$
\left\{\begin{array}{l}
\frac{d \phi}{d t}=-\phi+T_{n, \lambda}(\phi), \\
\phi=u \in W_{0}^{1, p}(\Omega) \backslash K .
\end{array}\right.
$$

Denote $\tau(u)$ to be the maximal interval of existence of $\phi_{u}(t)$.

Claim 1: $\phi_{u}(t)$ cannot enter $\mathcal{N}_{c, \varepsilon}$ before it enters $\mathcal{Q}_{n}^{c-\delta}$ for small $\delta$, large $n$ and $u \in \mathcal{A}_{n, 2 \varepsilon}^{c, \delta}$. 
Indeed, if the claim fails, then for every $\delta>0, \phi_{u}(t)$ will enter $\mathcal{N}_{c, \varepsilon}$ before it enters $\mathcal{Q}_{n}^{c-\delta}$. Since $u \in \mathcal{A}_{n, 2 \varepsilon}^{c, \delta} \subset W_{0}^{1, p}(\Omega) \backslash \mathcal{N}_{c, 2 \varepsilon}$, there exist $0 \leq t_{1}<t_{2}<\tau(u)$ such that $\phi_{u}(t) \in \mathcal{N}_{c, 2 \varepsilon} \backslash \mathcal{N}_{c, \varepsilon}$ for $t \in\left(t_{1}, t_{2}\right]$ and

$$
\operatorname{dist}\left(\phi_{u}\left(t_{1}\right), K_{c}^{*}\right)=2 \varepsilon, \quad \operatorname{dist}\left(\phi_{u}\left(t_{2}\right), K_{c}^{*}\right)=\varepsilon
$$

By [14, Lemma 2.1], $C\left\|u-S_{n, \lambda}(u)\right\|^{2}\left(\|u\|+\left\|S_{n, \lambda}(u)\right\|\right)^{p-2} \leq\left\langle I_{n}(u), u-T_{n, \lambda}(u)\right\rangle$. On the other hand, by the choice of $t_{1}$ and $t_{2}$, we know that $\phi_{u}(t) \in \mathcal{A}_{n, \varepsilon}^{c, \delta}$ for $t \in\left(t_{1}, t_{2}\right]$. Thanks to [17, Lemma 3.8], $\left\|u-S_{n, \lambda}(u)\right\| \geq\left(\frac{\gamma}{C}\right)^{1 /(p-1)}$ for large $n$. This, together with (2.6) and [14, Lemma 2.1], implies

$$
\begin{aligned}
\varepsilon & \leq\left\|\phi_{u}\left(t_{2}\right)-\phi_{u}\left(t_{1}\right)\right\| \leq \int_{t_{1}}^{t_{2}}\left\|\phi_{u}(t)-T_{n, \lambda}\left(\phi_{u}(t)\right)\right\| d t \\
& \leq C \int_{t_{1}}^{t_{2}}\left\|\phi_{u}(t)-S_{n, \lambda}\left(\phi_{u}(t)\right)\right\| d t \\
& \leq C \int_{t_{1}}^{t_{2}}\left\|\phi_{u}(t)-S_{n, \lambda}\left(\phi_{u}(t)\right)\right\|^{2}\left(\left\|\phi_{u}(t)\right\|+\left\|S_{n, \lambda}\left(\phi_{u}(t)\right)\right\|\right)^{p-2} d t \\
& \leq C \int_{t_{1}}^{t_{2}}\left\langle I_{n}\left(\phi_{u}(t)\right), \phi_{u}(t)-T_{n, \lambda}\left(\phi_{u}(t)\right)\right\rangle d t \\
& =C\left(I_{n}\left(t_{1}\right)-I_{n}\left(t_{2}\right)\right) \leq 4 C \delta .
\end{aligned}
$$

A contradiction with $\delta<4 C / \varepsilon$.

Claim 2: There exists $\tau_{1}(t)<\tau(u)$ such that $\phi_{u}\left(\tau_{1}(u)\right) \in \mathcal{Q}_{n}^{c-\delta}$ for large $n$ and $u \in \mathcal{A}_{n, 2 \varepsilon}^{c, \delta}$.

If the claim is not true, then $\phi_{u}(t) \in \mathcal{Q}_{n}^{c+\delta} \backslash \mathcal{Q}_{n}^{c-\delta}$ for all $t \in(0, \tau(u))$. We first consider the case of $\tau(u)<+\infty$. In fact, by Claim $1, \phi_{u}(t) \notin \mathcal{N}_{c, \varepsilon}$, i.e., $\phi_{u}(t) \in \mathcal{A}_{n, \varepsilon}^{c, \delta}$ for all $t \in(0, \tau(u))$. Since $\left\|I_{n}^{\prime}(u)\right\| \geq \gamma>0$ for $u \in \mathcal{A}_{n, \varepsilon}^{c, \delta}$ and large $n$, we must have

$$
\left\|\phi_{u}(t)\right\| \rightarrow \infty \quad \text { as } t \rightarrow \tau(u) .
$$

On the other hand, by [14, Lemma 2.1] and [17, Lemma 5.2], we have

$$
\begin{aligned}
\left\|\phi_{u}(t)-\phi_{u}(0)\right\| & \leq \int_{0}^{t}\left\|\phi_{u}(s)-T_{\lambda, n}\left(\phi_{u}(s)\right)\right\| d s \\
& \leq C \int_{0}^{t}\left\|\phi_{u}(s)-S_{\lambda, n}\left(\phi_{u}(s)\right)\right\| d s \\
& \leq C \int_{0}^{t}\left(1+\left\|\phi_{u}(s)-S_{\lambda, n}\left(\phi_{u}(s)\right)\right\|\right)^{p} d s \\
& \leq C \int_{0}^{t}\left(1+\left\|\phi_{u}(s)-S_{\lambda, n}\left(\phi_{u}(s)\right)\right\|\right)^{2}\left(\left\|\phi_{u}(s)\right\|+\left\|S_{\lambda, n}\left(\phi_{u}(s)\right)\right\|\right)^{p-2} d s \\
& \leq C \int_{0}^{t}\left\|\phi_{u}(s)-S_{\lambda, n}\left(\phi_{u}(s)\right)\right\|^{2}\left(\left\|\phi_{u}(s)\right\|+\left\|S_{\lambda, n}\left(\phi_{u}(s)\right)\right\|\right)^{p-2} d s \\
& \leq C\left(I_{n}\left(\phi_{u}(0)\right)-I_{n}\left(\phi_{u}(t)\right)\right) \leq C .
\end{aligned}
$$

This means $\left\|\phi_{u}(t)\right\| \leq\|u\|+C$ for all $t \in(0, \tau(u))$, which contradicts with (2.7). It follows that there must exist $\tau_{1}(u)<\tau(u)$ such that $\phi_{u}\left(\tau_{1}(u)\right) \in \mathcal{Q}_{n}^{c-\delta}$ for $u \in \mathcal{A}_{n, 2 \varepsilon}^{c, \delta}$, large $n$ and 
$\tau(u)<+\infty$. Next, we consider the case of $\tau(u)=+\infty$. Since $\left\|u-S_{n, \lambda}(u)\right\| \geq\left(\frac{\gamma}{C}\right)^{1 /(p-1)}$ for all $u \in \mathcal{A}_{n, \varepsilon}^{c, \delta}$ and large $n$, it follows from [14, Lemma 2.1] and [17, Lemma 5.2] that

$$
\begin{aligned}
\frac{d I_{n}\left(\phi_{u}(t)\right)}{d t} & =\left\langle I_{n}\left(\phi_{u}(t)\right),-\phi_{u}(t)+T_{n, \lambda}\left(\phi_{u}(t)\right)\right\rangle \\
& \leq-C\left\|\phi_{u}(t)-S_{n, \lambda}\left(\phi_{u}(t)\right)\right\|^{2}\left(\left\|\phi_{u}(t)\right\|+\left\|S_{n, \lambda}\left(\phi_{u}(t)\right)\right\|\right)^{p-2} \\
& \leq-C\left\|\phi_{u}(t)-S_{n, \lambda}\left(\phi_{u}(t)\right)\right\|^{2}\left(1+\left\|\phi_{u}(t)-S_{n, \lambda}\left(\phi_{u}(t)\right)\right\|\right)^{p-2} \\
& \leq-C<0 .
\end{aligned}
$$

Thus, there also exists $\tau_{1}(u) \in(0,+\infty)$ such that $\phi_{u}\left(\tau_{1}(u)\right) \in \mathcal{Q}_{n}^{c-\delta}$ for $u \in \mathcal{A}_{n, 2 \varepsilon}^{c, \delta}$ and $\tau(u)=$ $+\infty$. Moreover, we must have $\phi_{u}(t) \in \mathcal{Q}_{n}^{c-\delta}$ for $t \in\left(\tau_{1}(u), \tau(u)\right)$ since $\frac{d I_{n}\left(\phi_{u}(t)\right)}{d t} \leq 0$ for all $u \in W_{0}^{1, p}(\Omega) \backslash K$.

Let

$$
\eta_{n}(u)= \begin{cases}\phi_{u}\left(\tau_{1}(u)\right), & u \in \mathcal{A}_{n, 2 \varepsilon}^{c}, \\ u, & u \in \mathcal{Q}_{n}^{c-\delta} .\end{cases}
$$

Then, by the continuity of $\phi_{u}(t), \eta_{n}(u)$ is continuous. Note that $\phi_{u}(t)$ is odd and $\tau_{1}(u)$ is even, we see that $\eta_{n}(u)$ is odd and it is the desired map. The situation of $p \geq 2$ can be proved in a similar way. Therefore, we complete the proof of this lemma.

Proof of Theorem 1.1 We first consider the case $\lambda \geq \lambda_{1}$. Thanks to Lemma 2.1 and [9, Theorem 1.1], (1.1) has infinitely many sign-changing solutions. Next, we consider the case of $\lambda \in\left(0, \lambda_{1}\right)$. Since for every $n \in \mathbb{N}, 0 \leq d_{n, k} \leq d_{n, k+1}$ for all $k \in \mathbb{N}, d_{k} \leq d_{k+1}$ for all $k \in \mathbb{N}$. It follows that two cases may occur:

Case 1: There are $1<k_{1}<k_{2}<\cdots$ such that $d_{k_{1}}<d_{k_{2}}<\cdots$.

In this case, Problem (1.1) has infinitely many sign-changing solutions.

Case 2: There exists $k_{0}>0$ such that $d_{*}=d_{k}$ for all $k \geq k_{0}$.

In this case, if $\left(K^{*} \cap J^{d_{*}+\delta} \bigvee^{d_{*}-\delta}\right) \backslash K_{d_{*}}^{*} \neq \emptyset$ for every $\delta>0$ small enough, then Problem (1.1) also has infinitely many sign-changing solutions. Otherwise, there exists $\delta_{0}>0$ such that $\left(K^{*} \cap J^{d_{*}+\delta} \backslash \bigvee^{d_{*}-\delta}\right)=K_{d_{*}}^{*}$ for $\delta<\delta_{0}$. Thanks to Lemmas 2.4 and 2.5, there exists $\eta_{n}$ such that $\eta_{n}\left(\mathcal{A}_{n, 2 \varepsilon}^{d_{*}} \cup \mathcal{Q}_{n}^{d_{*}-\delta}\right) \subset \mathcal{Q}_{n}^{d_{*}-\delta}$ for small $\delta$ and large $n$. Fix $l \in \mathbb{N}$ and $k \geq k_{0}$, the definitions of $d_{k}$ and $d_{k+l}$ give that there exists a large $n$ such that $d_{n, k}>d_{*}-\delta$ and $d_{n, k+l}<d_{*}+\delta$ for small $\delta \in(0,1)$. By the definition of $d_{n, k+l}$, there exists $Z \in \Gamma_{k+l}$ such that $\sup _{Z} I_{n}(u)<d_{*}+\delta$, where $Z=h\left(B_{m} \backslash B\right) \backslash D_{\mu}, h \in G_{m}$ and gen $(B) \leq m-k-l$. It follows that $h\left(B_{m} \backslash B\right) \backslash \mathcal{N}_{d_{*}, 2 \varepsilon} \subset \mathcal{A}_{n, 2 \varepsilon}^{c, \delta} \cup$ $\mathcal{Q}_{n}^{c-\delta}$. Thus, $\eta_{n}\left(h\left(B_{m} \backslash B\right) \backslash \mathcal{N}_{d_{*}, 2 \varepsilon}\right) \subset \mathcal{Q}_{n}^{d_{*}-\delta}$. By the choice of $\delta$ and $B_{m}$, we have $\eta_{n} \circ h \in G_{m}$. If $\operatorname{gen}\left(B \cup h^{-1}\left(\mathcal{N}_{d_{*}, 2 \varepsilon}\right)\right) \leq m-k$, then we have

$$
d_{*}-\delta<d_{n, k} \leq \sup _{\eta_{n} \circ h\left(B_{m} \backslash\left(B \cup h^{-1}\left(\mathcal{N}_{d_{*}, 2 \varepsilon}\right)\right)\right)} I_{n}(u) \leq d_{*}-\delta .
$$

A contradiction. By the properties of gen, we have

$$
m-k+1 \leq \operatorname{gen}\left(B \cup h^{-1}\left(\mathcal{N}_{d_{*}, 2 \varepsilon}\right)\right) \leq \operatorname{gen}(B)+\operatorname{gen}\left(\mathcal{N}_{d_{*}, 2 \varepsilon}\right) \leq m-k-l+\operatorname{gen}\left(\mathcal{N}_{d_{*}, 2 \varepsilon}\right) .
$$

This implies gen $\left(\mathcal{N}_{d_{*}, 2 \varepsilon}\right) \geq l+1$. Since $l \in \mathbb{N}$ is arbitrary, we have $\operatorname{gen}\left(\mathcal{N}_{d_{*}, 2 \varepsilon}\right)=+\infty$, which contradicts with (2.5). 


\section{Competing interests}

The authors declare that they have no competing interests.

\section{Authors' contributions}

The authors typed, read and approved the final manuscript.

\section{Acknowledgements}

The work was supported by the Natural Science Foundation of China $(11071180,11171247)$ and College Postgraduate

Research and Innovation Project of Jiangsu Province (CXZZ110082).

\section{Received: 14 December 2012 Accepted: 28 May 2013 Published: 19 June 2013}

\section{References}

1. Brezis, H, Nirenberg, L: Positive solutions of nonlinear elliptic equations involving critical Sobolev exponent. Commun. Pure Appl. Math. 36, 437-478 (1983)

2. Cerami, G, Fortunato, D, Struwe, M: Bifurcation and multiplicity results for nonlinear elliptic problems involving critical Sobolev exponents. Ann. Inst. Henri Poincaré, Anal. Non Linéaire 1, 341-350 (1984)

3. Clapp, M, Weth, T: Multiple solutions for the Brezis-Nirenberg problem. Adv. Differ. Equ. 10, 463-480 (2005)

4. Devillanova, G, Solimini, S: Concentration estimates and multiple solutions to elliptic problems at critical growth. Adv. Differ. Equ. 7, 1257-1280 (2002)

5. Fortunato, D, Jannelli, E: Infinitely many solutions for some nonlinear elliptic problems in symmetrical domains. Proc. R. Soc. Edinb. A 105, 205-213 (1987)

6. Solimini, S: A note on compactness-type properties with respect to Lorenz norms of bounded subsets of a Sobolev spaces. Ann. Inst. Henri Poincaré, Anal. Non Linéaire 12, 319-337 (1995)

7. Schechter, M, Zou, W: On the Brézis-Nirenberg problem. Arch. Ration. Mech. Anal. 197, 337-356 (2010)

8. Alves, C, Ding, Y: Multiplicity of positive solutions to a $p$-Laplacian equation involving critical nonlinearity. J. Math. Anal. Appl. 279, 508-521 (2003)

9. Cao, D, Peng, S, Yan, S: Infinitely many solutions for $p$-Laplacian equation involving critical Sobolev growth. J. Funct. Anal. 262, 2861-2902 (2012)

10. Cingolani, S, Vannella, G: Multiple positive solutions for a critical quasilinear equation via Morse theory. Ann. Inst. Henri Poincaré, Anal. Non Linéaire 26, 397-413 (2009)

11. Degiovanni, $M$, Lancelotti, S: Linking solutions for $p$-Laplace equations with nonlinearity at critical growth. J. Funct. Anal. 256, 3643-3659 (2009)

12. Allegretto, W, Huang, Y: A Picone's identity for the $p$-Laplacian and applications. Nonlinear Anal. 32, $819-830$ (1998)

13. Iturriaga, L, Massa, E, Sanchez, J, Ubilla, P: Positive solutions of the $p$-Laplacian involving a superlinear nonlinearity with zeros. J. Differ. Equ. 248, 309-327 (2010)

14. Bartsch, T, Liu, Z, Weth, T: Nodal solutions of $p$-Laplacian equation. Proc. Lond. Math. Soc. 91, 129-152 (2005)

15. Lindqvist, P: On the equation $\operatorname{div}\left(|\nabla u|^{p-2} \nabla u\right)+\lambda|u|^{p-2} u=0$. Proc. Am. Math. Soc. 109, 157-164 (1990)

16. Cuesta, M: Eigenvalue problem for the $p$-Laplacian with indefinite weights. Electron. J. Differ. Equ. 2001, 1-9 (2001)

17. Bartsch, T, Liu, Z: On a superlinear elliptic p-Laplacian equation. J. Differ. Equ. 198, 149-175 (2004)

18. Tolksdorf, P: Regularity for a more general class of quasilinear elliptic equations. J. Differ. Equ. 51, 126-150 (1984)

19. Rabinowitz, P: Minimax Methods in Critical Point Theory with Applications to Differential Equations. CBMS Reg. Conf. Ser. Math., vol. 65. Am. Math. Soc., Providence (1986)

\section{Submit your manuscript to a SpringerOpen ${ }^{\ominus}$ journal and benefit from:}

- Convenient online submission

Rigorous peer review

- Immediate publication on acceptance

- Open access: articles freely available online

- High visibility within the field

- Retaining the copyright to your article 